\title{
Why drug approval needs better evidence
}

\author{
Fiona Godlee editor in chief
}

The BMJ

It's good to see new NICE guidance on major trauma. Our summary of it (doi:10.1136/bmj.i3051) focuses on assessment of patients with major trauma and management of patients who are actively bleeding. It carries some new take home messages: avoid preliminary imaging when patients need immediate computed tomography; allow "permissive hypotension" in patients who are actively bleeding; and use blood rather than crystalloids or colloids.

The evidence base for managing trauma hasn't always been good and in some areas remains controversial: think of the continuing bitter debate over crystalloid versus colloid (doi:10. 1136/bmj.h1656, doi:10.1136/bmj.i1027). But the evidence has improved in quantity and confidence over recent years.

Not so for rare diseases. Efforts to develop treatments for these conditions are bedevilled by low return on investment and difficulty recruiting patients for large enough trials. Drugs tend to be approved on the basis of small, short term, placebo controlled trials that use surrogate endpoints. Regulators justify this leniency on the understanding that manufacturers will follow up with post-marketing studies. But does this happen, and what's the result?

Roberta Joppi and colleagues reviewed the cumulative evidence for all six "orphan" products approved by the European
Medicines Agency in 2004 (doi:10.1136/bmj.i2978). They found limited evidence at approval, which post-marketing research over the ensuing 10 years did little to improve on. In most cases the drugs performed no better or worse than comparators, but the original regulatory decisions were not revised, and manufacturers weren't obliged to carry out further studies.

The authors say that the problem is not limited to rare conditions, as Huseyin Naci and colleagues found last year when they looked at drug approvals for glucose lowering in type 2 diabetes (doi:10.1136/bmj.h5260). This lucrative mass market has created a gold rush for "me too" treatments, with over 200 molecules in the development pipeline. But Naci and colleagues noted an equally growing list of drug withdrawals and black box warnings that should curb regulatory enthusiasm for "timely market access," they said.

Both articles conclude that licensing agencies urgently need to demand higher standards of evidence before and after approval. Higher costs of evaluation should be more than offset by less spending on ineffective treatments, with better outcomes and fewer harms to patients. In the meantime, patients and their doctors need honest and open communication about the true strength of the evidence behind licensing decisions. 